\title{
The herbicide ketoclomazone inhibits 1-deoxy- D-xylulose 5-phosphate synthase in the 2-C-methyl- D-erythritol 4-phosphate pathway and shows antibacterial activity against Haemophilus influenzae
}

\author{
Yukiko Matsue $^{1}$, Hiroko Mizuno ${ }^{1}$, Takeo Tomita ${ }^{1}$, Tadao Asami $^{2,3}$, Makoto Nishiyama ${ }^{1}$ \\ and Tomohisa Kuzuyama ${ }^{1}$
}

Two distinct metabolic pathways have been elucidated for the formation of isopentenyl diphosphate and dimethylallyl diphosphate, essential metabolic precursors for isoprenoid biosynthesis: the mevalonate pathway, found ubiquitously in mammals, and the 2-C-methyl-D-erythritol 4-phosphate (MEP) pathway, found in most bacteria. As the MEP pathway is absent from mammals, all MEP pathway enzymes represent effective targets for the development of antibacterial drugs. In this study, we found that a herbicide, ketoclomazone, exhibited antibacterial activity against a pathogenic bacterium, Haemophilus influenzae, with an MIC value of $12.5 \mu \mathrm{g} \mathrm{ml}^{-1}$ and that antibacterial activity was suppressed by adding 1-deoxyxylulose, a free alcohol of 1-deoxy-D-xylulose 5-phosphate (DXP). DXP is an MEP pathway intermediate synthesized from pyruvate and D-glyceraldehyde 3-phosphate (D-GAP) by the action of DXP synthase. Thus, we investigated the enzyme kinetics of DXP synthase of $H$. influenzae (HiDXS) to elucidate an inhibitory mechanism of ketoclomazone on HiDXS. The dxs gene was cloned from $H$. influenzae and overexpressed in Escherichia coli, and the enzyme was purified to homogeneity. The purified HiDXS was a soluble dimeric 70-kDa protein. Steady-state kinetic constants for HiDXS were calculated, and Lineweaver-Burk plots were consistent with a ping-pong bi bi mechanism. The kinetics of inhibition by ketoclomazone suggested that ketoclomazone binds to an unidentified inhibitor-binding site that differs from both the pyruvate-binding site and the D-GAP-binding site on DXP synthase. These data reveal the inhibitory mechanism of ketoclomazone on DXP synthase.

The Journal of Antibiotics (2010) 63, 583-588; doi:10.1038/ja.2010.100; published online 1 September 2010

Keywords: inhibitor; IPP; terpenoid

\section{INTRODUCTION}

Isoprenoids, molecules found in all organisms, are important for the production of steroid hormones in mammals, carotenoids in plants, and ubiquinone or menaquinone and lipid intermediates for peptidoglycan biosynthesis in bacteria. ${ }^{1}$ All isoprenoids are synthesized by consecutive condensations of a five-carbon monomer, isopentenyl diphosphate (IPP), to its isomer dimethylallyl diphosphate, both of which are essential metabolic precursors for isoprenoid biosynthesis.

Two distinct metabolic pathways exist for IPP and dimethylallyl diphosphate biosynthesis: the classical mevalonate pathway found ubiquitously in mammals and Archaebacteria, and the recently discovered 2-C-methyl-D-erythritol 4-phosphate (MEP) pathway (Figure 1). ${ }^{2,3}$ The MEP pathway consists of seven enzymes. The initial step of this pathway is the formation of 1-deoxy-D-xylulose 5-phosphate (DXP) by a transketolase-type condensation of pyruvate and D-glyceraldehyde 3-phosphate (D-GAP), catalyzed by DXP synthase in a thiamine diphosphate-dependent manner. ${ }^{4-6}$ This enzyme is also essential for the synthesis of vitamins $B_{1}$ and $B_{6}$ in bacteria. ${ }^{6}$ In the second step, intramolecular rearrangement and reduction of DXP simultaneously occur to form MEP. ${ }^{7}$ This reaction is catalyzed by NADPH-dependent DXP reductoisomerase. ${ }^{8}$ MEP is finally converted into both IPP and dimethylallyl diphosphate by the action of the following five enzymes, MEP cytidylyltransferase, ${ }^{9,10} 4$-(cytidine $5^{\prime}$-diphospho)-2-C-methyl-D-erythritol kinase, ${ }^{11,12}$ 2-C-methyl-D-erythritol 2,4-cyclodiphosphate synthase, ${ }^{13,14}$ 4-hydroxy3-methylbut-2-en-1-yl diphosphate synthase ${ }^{15}$ and 4-hydroxy-3methylbut-2-en-1-yl diphosphate reductase. ${ }^{16}$

The MEP pathway is present in most bacteria, including pathogens, as well as in plant chloroplasts, and the apicoplasts of Apicomplexan protozoa such as Plasmodium and Toxoplasma. ${ }^{3}$ As the MEP pathway is absent from mammals, all MEP pathway enzymes represent effective targets for the development of antibacterial and antimalarial drugs and

\footnotetext{
${ }^{1}$ Biotechnology Research Center, The University of Tokyo, Tokyo, Japan and ${ }^{2}$ RIKEN, Advanced Science Institute, Saitama, Japan

${ }^{3}$ Current address: Graduate School of Agricultural and Life Sciences, The University of Tokyo, 1-1-1 Yayoi, Bunkyo-ku, Tokyo 113-8657, Japan.

Correspondence: Dr T Kuzuyama, Biotechnology Research Center, The University of Tokyo, 1-1-1 Yayoi, Bunkyo-ku, Tokyo 113-8657, Japan.

E-mail: utkuz@mail.ecc.u-tokyo.ac.jp

Received 24 June 2010; revised 26 July 2010; accepted 1 August 2010; published online 1 September 2010
} 

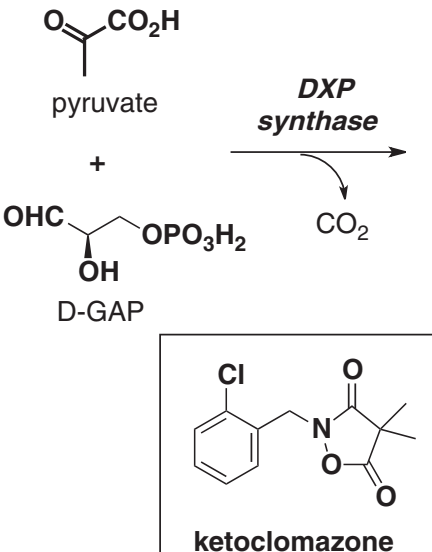

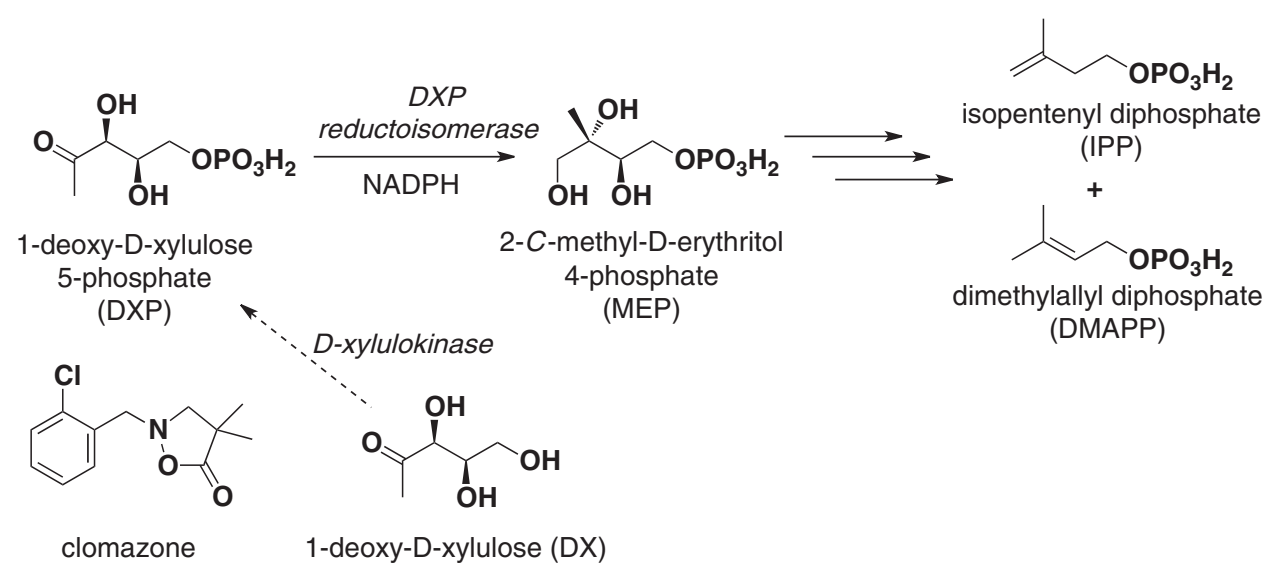

Figure 1 1-Deoxy-D-xylulose 5-phosphate (DXP) synthase reaction in the 2-C-methyl-D-erythritol 4-phosphate (MEP) pathway for isopentenyl diphosphate (IPP) and dimethylallyl diphosphate (DMAPP) biosynthesis.

herbicides. In fact, we have demonstrated that in Escherichia coli the knock-out mutant of DXP reductoisomerase is lethal, ${ }^{17}$ and that fosmidomycin is a potent and specific inhibitor of the enzyme. ${ }^{18}$ Jomaa et al. ${ }^{19}$ reported that fosmidomycin and its derivative inhibited DXP reductoisomerase of Plasmodium falciparum, and that these inhibitors cured mice infected with the rodent malaria parasite Plasmodium vinckei. Thus, DXP reductoisomerase has been shown to represent an effective target for the development of antibacterial and antimalarial drugs.

Ketoclomazone (2-(2-chlorobenzyl)-4,4-dimethyl-isoxazolidine-3,5dione) is a derivative of a soil-applied herbicide, clomazone, also known as dimethazone or FMC 57020. ${ }^{20}$ It has been reported that clomazone and ketoclomazone cause significantly lower chlorophyll and carotenoid levels in barley leaves. ${ }^{21}$ By contrast, other studies have suggested that ketoclomazone, but not clomazone, inhibits DXP synthase activity in vitro. Lichtenthaler and coworkers ${ }^{22}$ reported that ketoclomazone, but not clomazone, inhibited the activity of a recombinant DXP synthase from Chlamydomonas, with an $\mathrm{IC}_{50}$ value of $0.1 \mathrm{~mm}$. In addition, Ferhatoglu and Barrett demonstrated that $20 \mu \mathrm{M}$ ketoclomazone inhibited $47 \%$ of DXP synthase activity in crude extracts from E. coli expressing a plant Catharanthus roseus DXP synthase gene. ${ }^{23}$ These reports have suggested that clomazone may be converted into ketoclomazone in plants to inhibit DXP synthase. However, a mechanism of inhibition by ketoclomazone of DXP synthase has not been elucidated, although clomazone and ketoclomazone have been used as chemical tools to suppress the MEP pathway in plants. ${ }^{24-26}$ In addition, antibacterial activities of clomazone and ketoclomazone have not been reported, although most bacteria, including pathogens, utilize the MEP pathway for isoprenoid biosynthesis. ${ }^{27}$

In this study, we found that ketoclomazone inhibited the growth of E. coli and Haemophilus influenzae, and that this inhibition was suppressed by the addition of 1-deoxyxylulose (DX), a free alcohol of DXP. This finding strongly suggests that a target of ketoclomazone is DXP synthase in the MEP pathway, leading us to evaluate ketoclomazone as an inhibitor of DXP synthases from the bacteria. This evaluation revealed for the first time that ketoclomazone binds to the site of inhibition, which differs from both the pyruvate-binding site and the D-GAPbinding site on DXP synthase. These data are the first demonstration that the antibacterial activity of ketoclomazone against E. coli and $H$. influenzae is due to the inhibition of DXP synthase activity by ketoclomazone. This study may lead to the development of new antibacterial drugs through the chemical derivatization of ketoclomazone.

\section{MATERIALS AND METHODS}

\section{Materials}

Genomic DNA from H. influenzae ATCC51907D, which was used in the bacterial sequencing project, ${ }^{28}$ was purchased from the American Type Culture Collection (Manassas, VA, USA). Ketoclomazone was synthesized according to Sakakibara $e t$ al. ${ }^{24}$ The compound DX was synthesized according to Kennedy et al. ${ }^{29}$ DL-GAP, pyruvate and NADPH were purchased from Sigma-Aldrich (Tokyo, Japan). Thiamine diphosphate was purchased from Wako Pure Chemical (Osaka, Japan).

\section{MIC values against $H$. influenzae and $E$. coli}

H. influenzae ATCC43095 and E. coli W3110 were cultivated on plates of Bacto Brain Heart Infusion agar (Becton, Dickinson and Company, Starks, MD, USA) at $35^{\circ} \mathrm{C}$ for $20 \mathrm{~h}$. Well-separated colonies were then suspended in the following liquid media: $21 \mathrm{gl}^{-1}$ Difco Mueller Hinton broth, $5 \mathrm{gl}^{-1}$ Bacto Yeast extract, $15 \mathrm{mgl}^{-1}$ Hematin (Sigma-Aldrich), $12.5 \mathrm{mgl}^{-1} \mathrm{MgCl}_{2}, 25 \mathrm{mgl}^{-1} \mathrm{CaCl}_{2}$ and $15 \mathrm{mgl}^{-1} \beta$-NAD for $H$. influenzae; and $21 \mathrm{gl}^{-1}$ Difco Mueller Hinton broth, $12.5 \mathrm{mgl}^{-1} \mathrm{MgCl}_{2}$ and $25 \mathrm{mgl}^{-1} \mathrm{CaCl}_{2}$ for E. coli. Optical density at $620 \mathrm{~nm}$ $\left(\mathrm{OD}_{620 \mathrm{~nm}}\right)$ of the cell suspensions was adjusted to 0.5. The adjusted cell suspensions were diluted 1:600 ratio by adding the media described above. Aliquots $(95 \mu \mathrm{l})$ of the diluted cell suspensions were inoculated into each well of 96-well plates. To each well, $5 \mu \mathrm{l}$ of ketoclomazone dissolved in dimethyl sulfoxide was added to give final concentrations of $0.39,0.78,1.56,3.12$, $6.25,12.5,25,50,100,200,400$ and $800 \mu \mathrm{g} \mathrm{ml}^{-1}$. Ampicillin $(0.10,0.19,0.39$, $0.78,1.56,3.12,6.25$ and $\left.12.5 \mu \mathrm{g} \mathrm{ml}^{-1}\right)$ and fosmidomycin $(0.10,0.19,0.39$, $0.78,1.56,3.12,6.25$ and $12.5 \mu \mathrm{g} \mathrm{ml}^{-1}$ ) were used as controls. H. influenzae and E. coli were incubated at $35^{\circ} \mathrm{C}$ for $20 \mathrm{~h}$. The MIC was read as the lowest concentration with no visible growth.

\section{Construction of the Gateway-adapted destination plasmid for} high-level expression of the histidine-tagged recombinant HiDXS in E. coli

In the genomic database of $H$. influenzae (accession number L42023), HI1439 has been identified as DXP synthase. On the basis of the entire nucleotide sequence of HI1439, the oligonucleotide forward primer, pHiDXS-N, 5'-CACCATGACTAACAATATGAACAATTATCC-3' (start codon underlined) and the reverse primer, pHiDXS-C, $5^{\prime}$-TAAATTACCTTGTTTTGCAATAAAG TTGAG-3' were synthesized (Operon Biotechnologies, Tokyo, Japan) and used together with total DNA from $H$. influenzae ATCC51907D to amplify the HI1439 gene. Using iProof high-fidelity DNA polymerase (Bio-Rad Laboratories, Tokyo, Japan), a 1.9-kb fragment was amplified. The PCR-amplified DNA fragment was cloned into a pENTR/D-TOPO vector (Invitrogen, Tokyo, Japan) by a BR recombination reaction to generate an entry clone, pENTRHiDXS. Automated nucleotide sequencing confirmed the fidelity of the PCR product (Shimadzu Biotech, Kyoto, Japan). An LR recombination reaction between pENTR-HiDXS and a destination vector, pDEST17 (Invitrogen), was 
performed to generate an expression plasmid, pDEST17-HiDXS, using the protocol recommended by the supplier (Invitrogen).

\section{Expression and purification of the recombinant HiDXS}

E. coli BL21(DE3) (Takara Bio, Otsu, Japan) was used as the host for the expression of HiDXS as a hexahistidine-tagged protein. Cultures of BL21(DE3)/ pDEST17-HiDXS were grown at $37^{\circ} \mathrm{C}$ in Terrific broth containing $50 \mu \mathrm{g} \mathrm{ml}^{-1}$ ampicillin until $\mathrm{OD}_{600 \mathrm{~nm}}=1.5$. After induction with $0.1 \mathrm{~mm}$ isopropyl- $\beta$-Dthiogalactopyranoside, the culture was grown at $18^{\circ} \mathrm{C}$ for $14 \mathrm{~h}$. Cells were harvested, and resuspended in a lysis buffer consisting of $50 \mathrm{~mm}$ Tris- $\mathrm{HCl}(\mathrm{pH}$ $8.0), 500 \mathrm{~mm} \mathrm{NaCl}, 60 \mathrm{~mm}$ imidazole ( $\mathrm{pH} 8.0), 20 \%(\mathrm{v} / \mathrm{v})$ glycerol and $1 \%(\mathrm{v} / \mathrm{v})$ Tween-20. After sonication with a Branson Sonifier 250 (Emerson, Tokyo, Japan) and centrifugation, the supernatant was passed over a $\mathrm{Ni}^{2+}$-nitrilotriacetic acid agarose column (Qiagen, Tokyo, Japan). The column was washed with 10-bed volumes of a wash buffer consisting of $50 \mathrm{~mm}$ Tris- $\mathrm{HCl}(\mathrm{pH} 8.0)$, $500 \mathrm{~mm} \mathrm{NaCl}, 60 \mathrm{~mm}$ imidazole $(\mathrm{pH} 8.0)$ and $20 \%(\mathrm{v} / \mathrm{v})$ glycerol. The His $6^{-}$ tagged protein was eluted with wash buffer containing $250 \mathrm{~mm}$ imidazole $(\mathrm{pH}$ 8.0). The eluate was dialyzed for $16 \mathrm{~h}$ at $4{ }^{\circ} \mathrm{C}$ against a buffer consisting of $50 \mathrm{~mm}$ Tris- $\mathrm{HCl}(\mathrm{pH} 8.0), 100 \mathrm{~mm} \mathrm{NaCl}$ and $10 \mathrm{~mm} \beta$-mercaptoethanol. Gel filtration chromatography on a Superdex-200 (GE Healthcare, Tokyo, Japan) fast protein LC column equilibrated with $25 \mathrm{~mm}$ HEPES (pH 7.5), $100 \mathrm{~mm}$ $\mathrm{NaCl}$ and $5 \mathrm{~mm}$ dithiothreitol was the final purification step. Fractions containing $H$. influenzae DXP synthase (HiDXS) were pooled, concentrated to $25 \mathrm{mg} \mathrm{ml}^{-1}$ by ultrafiltration with a Vivaspin 20 (30000 MWCO; Sartorius Stedim Biotech, Goettingen, Germany) and stored at $-80^{\circ} \mathrm{C}$.

\section{DXP synthase assay}

A spectrophotometric DXP synthase assay was employed using a coupled system with DXP reductoisomerase as previously described, ${ }^{30}$ with a slight modification. The assay system consisted of $40 \mathrm{~mm}$ Tris- $\mathrm{HCl}$ ( $\mathrm{pH} 7.5), 100 \mathrm{~mm}$ $\mathrm{NaCl}, 1 \mathrm{~mm} \mathrm{MgCl}_{2}, 1 \mathrm{~mm}$ dithiothreitol, $0.3 \mathrm{~mm} \mathrm{TPP}, 0.15 \mathrm{~mm} \mathrm{NADPH}$, pyruvate and DL-GAP, and $0.1 \mathrm{U}$ of E. coli DXP reductoisomerase in a final volume of $1 \mathrm{ml}$. The assay mixture was preincubated at $37^{\circ} \mathrm{C}$ for $3 \mathrm{~min}$, and the reaction was initiated by adding DXP synthase. The enzyme-dependent oxidation of NADPH was monitored in a spectrophotometer UV-160 equipped with a cell holder, CPS-240A (Shimadzu Biotech), adjusted to $37^{\circ} \mathrm{C}$. Initial velocities were determined from the slope of a plot of NADPH consumption versus incubation time. The molar extinction coefficient $(\varepsilon)$ of NADPH is $6220 \mathrm{M}^{-1} \mathrm{~cm}^{-1}$ at $340 \mathrm{~nm}$. One unit (U) of DXP synthase and DXP reductoisomerase is defined as the amount of the enzyme that causes oxidation of $1 \mu \mathrm{mol}$ of NADPH per min. As L-GAP would not affect DXS activity, racemic DL-GAP was used as the substrate of DXP synthase. The concentration of D-GAP is calculated as half of the DL-GAP concentration. Set concentrations of pyruvate $(0.1,0.15,0.2,0.4$ or $0.5 \mathrm{mM})$ and DL-GAP $(0.2,0.3,0.4,0.8$ or $1.0 \mathrm{mM})$ were used for HiDXS. Set concentrations of pyruvate $(0.025,0.03,0.05$ or $0.075 \mathrm{~mm})$ and DL-GAP $(0.3,0.4,0.6$ or $1.0 \mathrm{mM})$ were used for E. coli DXP synthase (EcDXS). EcDXS was prepared as described previously. ${ }^{31}$

\section{Equation for the analysis of kinetic data}

Steady-state kinetic parameters were calculated using SigmaPlot 10.0 and Enzyme Kinetics Module 1.3 (Systat Software, Point Richmond, CA, USA). The initial velocities for HiDXS measured at various concentrations of pyruvate and D-GAP were fit to Equation (1), where $[A]$ and $[B]$ are the concentrations of pyruvate and D-GAP, respectively, $K_{\mathrm{m}}^{\text {pyruvate }}$ and $K_{\mathrm{m}}^{\mathrm{D}-\mathrm{GAP}}$ are the MichaelisMenten constants for pyruvate and D-GAP at saturating second substrate concentrations, respectively, where $v$ is the velocity of DXP formation and $V_{\max }$ is the maximal velocity of DXP formation.

$$
v=V_{\max }[A][B] /\left(K_{\mathrm{m}}^{\text {pyruvate }}[B]+K_{\mathrm{m}}^{\mathrm{D}-\mathrm{GAP}}[A]+[A][B]\right)
$$

\section{DXP synthase inhibition assays}

In DXP synthase inhibition assays, ketoclomazone was preincubated at $37^{\circ} \mathrm{C}$ for $3 \mathrm{~min}$ in a reaction mixture that consisted of $40 \mathrm{~mm}$ Tris- $\mathrm{HCl}(\mathrm{pH} 7.5)$, $100 \mathrm{~mm} \mathrm{NaCl}, 1 \mathrm{~mm} \mathrm{MgCl}$, 1 mм dithiothreitol, $0.3 \mathrm{~mm}$ TPP, $0.15 \mathrm{~mm}$ NADPH, pyruvate, GAP and $0.1 \mathrm{U}$ of E. coli DXP reductoisomerase. The reaction was initiated by adding DXP synthase. For the HiDXS reaction, the concentrations of pyruvate and D-GAP were $200 \mu \mathrm{M}\left(\approx K_{\mathrm{m}}^{\text {pyruvate }}\right.$ for HiDXS $)$ and $200 \mu \mathrm{m}$
( $\approx K_{\mathrm{m}}^{\mathrm{D}-\mathrm{GAP}}$ for HiDXS), respectively. For the EcDXS reaction, the concentrations of pyruvate and D-GAP were $50 \mu \mathrm{M}\left(\approx K_{\mathrm{m}}^{\text {pyruvate }}\right.$ for EcDXS $)$ and $375 \mu \mathrm{M}$ $\left(\approx K_{\mathrm{m}}^{\mathrm{D}-\mathrm{GAP}}\right.$ for EcDXS), respectively. Dissociation constants $\left(K_{\mathrm{i}}\right)$ of the inhibitors for binding to HiDXS and EcDXS were calculated from secondary plots of inhibitor concentrations versus the intercept or the slope of the corresponding Lineweaver-Burk plots.

\section{RESULTS}

\section{MIC values for $E$. coli and $H$. influenzae}

First, we investigated the MIC of ketoclomazone for $E$. coli and $H$. influenzae in the absence or presence of $0.1 \% \mathrm{DX}$, a free alcohol of DXP. An intermediate in an early step in the MEP pathway, DXP is also a reaction product of DXP synthase (Figure 1). Ketoclomazone showed antibacterial activity against $E$. coli and $H$. influenzae with MIC values of 800 and $12.5 \mu \mathrm{g} \mathrm{ml}^{-1}$, respectively, in the absence of DX (Table 1). Importantly, the inhibitory effect of ketoclomazone was suppressed by the addition of $0.1 \% \mathrm{DX}$ to the growth medium. As DX has been reported to be converted into DXP by the action of D-xylulokinase and incorporated into the MEP pathway for IPP biosynthesis in E. coli (Figure 1), ${ }^{32}$ DX should be incorporated into the MEP pathway in $H$. influenzae as well. Therefore, the suppression caused by the addition of DX strongly suggested that ketoclomazone inhibited DXP synthases of E. coli and H. influenzae. Thus, we decided to evaluate ketoclomazone as an inhibitor of EcDXS and HiDXS.

\section{Properties of HiDXS overexpressed in $E$. coli}

To characterize DXP synthase of $H$. influenzae, we overexpressed the HiDXS gene in E. coli. The expression of HiDXS in E. coli was efficient, and the recombinant HiDXS was purified to homogeneity as a soluble protein. The purified HiDXS showed a single band on SDS polyacrylamide gel electrophoresis with a subunit molecular mass of $70 \mathrm{kDa}$ (Supplementary Figure S1). By gel filtration chromatography, the molecular mass of the enzyme was estimated to be $130 \mathrm{kDa}$. These results suggest that HiDXS is likely to be a dimer.

\section{Steady-state kinetic studies}

We calculated steady-state kinetic data for the purified HiDXS. Initial velocities for HiDXS were measured using a spectrophotometric assay that quantifies product formation by the concomitant oxidation of NADPH. Lineweaver-Burk plots (1/initial velocity versus $1 /$ concentration) of pyruvate and D-GAP were created (Figure 2 ). The four lines in both plots are essentially parallel for substrate concentrations between approximately $0.5 K_{\mathrm{m}}$ and approximately $2.5 K_{\mathrm{m}}$. These plots indicate the ping-pong bi bi mechanism of DXP synthase, in which the formation of a HiDXS-pyruvate complex and the dissociation of $\mathrm{CO}_{2}$ occur before DXP formation. Steady-state kinetic constants for HiDXS

Table 1 MIC values of ketoclomazone and other antibiotics against E. coli W3110 and $H$. influenzae ATCC43095

\begin{tabular}{lcccc}
\hline & \multicolumn{4}{c}{$\left.M I C(\mu g)^{-1}\right)$} \\
\cline { 2 - 5 } & \multicolumn{2}{c}{ E. coli } & \multicolumn{2}{c}{ H. influenzae } \\
\cline { 2 - 5 } & $-D X$ & $+D X^{a}$ & $-D X$ & $+D X^{a}$ \\
\hline Ketoclomazone $^{b}$ & 800 & $>800$ & 12.5 & $>50$ \\
Fosmidomycin & 6.25 & 6.25 & 0.78 & 0.78 \\
Ampicillin & 3.12 & 3.12 & 0.19 & 0.19
\end{tabular}

Abbreviations: DX, 1-deoxyxylulose; DXP, 1-deoxy-D-xylulose 5-phosphate; E. coli, Escherichia coli: $H$. influenzae, Haemophilus influenzae

$0.1 \%$ DX, a free alcohol of DXP, was added to the culture media

bThe maximum concentration investigated against $H$. influenzae was $50 \mu \mathrm{g} \mathrm{ml}^{-1}$. 

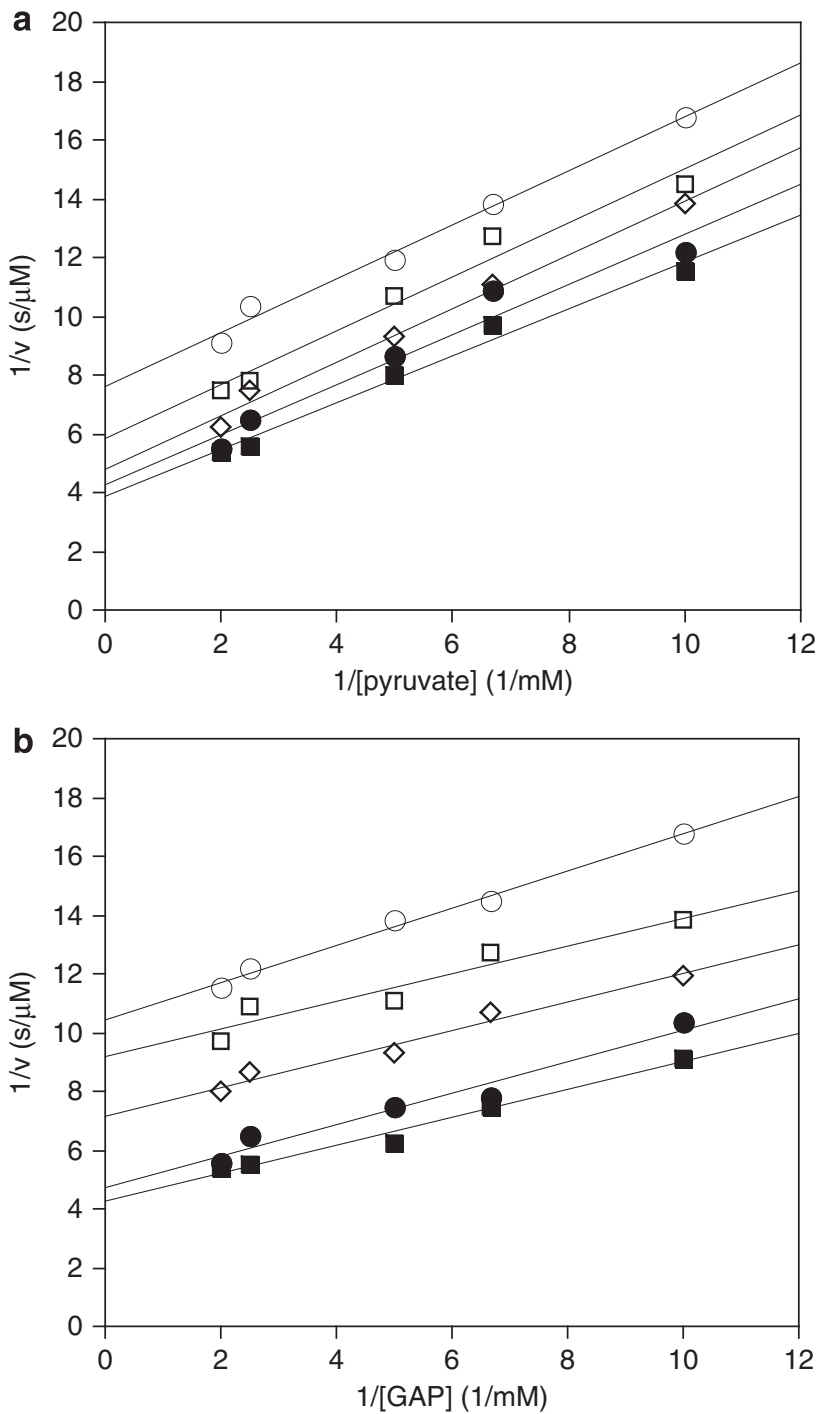

Figure 2 Kinetic analysis of $H$. influenzae 1-deoxy-D-xylulose 5-phosphate (DXP) synthase. (a) Lineweaver-Burk plot of initial-reaction velocities versus pyruvate concentrations at fixed concentrations of $D$-glyceraldehyde 3-phosphate (D-GAP). The concentration of pyruvate was varied between 0.1 and $0.5 \mathrm{~mm}$ at five D-GAP concentrations: $0.1 \mathrm{~mm}$, filled square; $0.15 \mathrm{~mm}$, filled circle; $0.2 \mathrm{~mm}$, diamond; $0.4 \mathrm{~mm}$, square; and $0.5 \mathrm{~mm}$, circle. (b) Lineweaver-Burk plot of initial-reaction velocities versus D-GAP concentrations at fixed concentrations of pyruvate. The concentration of D-GAP was varied between 0.1 and $0.5 \mathrm{~mm}$ at five pyruvate concentrations: $0.1 \mathrm{~mm}$, filled square; $0.15 \mathrm{~mm}$, filled circle; $0.2 \mathrm{~mm}$, diamond; $0.4 \mathrm{~mm}$, square; and $0.5 \mathrm{~mm}$, circle.

with pyruvate and D-GAP as substrates were determined by fitting initial velocity versus concentrations of pyruvate and D-GAP to the Michaelis-Menten equation (Equation (1)): $K_{\mathrm{m}}^{\text {pyruvate }}=190 \pm 20 \mu \mathrm{M}$, $K_{\mathrm{m}}^{\mathrm{D}-\mathrm{GAP}}=190 \pm 20 \mu \mathrm{M}$ and $V_{\max }=1.4 \pm 0.1 \mathrm{U}$ per mg protein. An apparent $K_{\mathrm{m}}^{\mathrm{TPP}}$ value for TPP was estimated to be $17 \pm 5 \mu \mathrm{M}$ at fixed concentrations of pyruvate $\left(200 \mu \mathrm{M}, \approx K_{\mathrm{m}}^{\text {pyruvate }}\right)$ and D-GAP $(200 \mu \mathrm{M}$, $\left.\approx K_{\mathrm{m}}^{\mathrm{D}-\mathrm{GAP}}\right)$. In this assay condition, TPP inhibited DXP synthase activity at $500 \mu \mathrm{M}$. We used $300 \mu \mathrm{M}$ TPP in all further DXP synthase assays because $300 \mu \mathrm{M}$ TPP afforded the maximum initial velocity.

To compare the kinetic constants of HiDXS with those of EcDXS, we calculated the kinetic constants of EcDXS using the same spectrophotometric assay used for HiDXS: $K_{\mathrm{m}}^{\text {pyruvate }}=48 \pm 14 \mu \mathrm{M}, K_{\mathrm{m}}^{\mathrm{D}-\mathrm{GAP}}$ $=370 \pm 110 \mu \mathrm{M}$ and $V_{\max }=3.5 \pm 0.8 \mathrm{U}$ per mg protein.

\section{Kinetic studies with ketoclomazone as an inhibitor}

We evaluated the performance of ketoclomazone as an inhibitor of HiDXS. A Lineweaver-Burk plot of the initial velocities for various concentrations of pyruvate and a fixed concentration of D-GAP $\left(200 \mu \mathrm{M}, \approx K_{\mathrm{m}}\right.$ for HiDXS) at different concentrations of ketoclomazone is shown in Figure 3a. The four lines in the plot are essentially parallel, indicating that the kinetics of inhibition is uncompetitive with respect to pyruvate. A $K_{\mathrm{i}}{ }^{\text {pyruvate }}$ value was calculated to be $28 \mu \mathrm{M}$ from a secondary plot (Figure $3 b$ ) of the Lineweaver-Burk plot (Figure 3a). In addition, a Lineweaver-Burk plot of the initial velocities for various concentrations of D-GAP and a fixed concentration of pyruvate $\left(200 \mu \mathrm{M}, \approx K_{\mathrm{m}}\right)$ at different concentrations of ketoclomazone is shown in Figure 3c. The four lines in the plot intersect near the horizontal axis, indicating that the kinetics of inhibition is mixed type with respect to D-GAP. $K_{\mathrm{m}}^{\mathrm{D}-\mathrm{GAP}}$ and $K_{\mathrm{i}}^{{ }^{\prime}}$-GAP values were almost equal and calculated to be 22 and $23 \mu \mathrm{M}$, respectively, from a secondary plot (Figure 3d) of the Lineweaver-Burk plot (Figure 3c).

We calculated dissociation constants of ketoclomazone for binding to EcDXS to compare the constants with those of HiDXS. A Lineweaver-Burk plot of the initial velocities for various concentrations of pyruvate and a fixed concentration of D-GAP $\left(375 \mu \mathrm{M}, \approx K_{\mathrm{m}}\right.$ for EcDXS) at different concentrations of ketoclomazone is shown in Supplementary Figure S2A. As is the case with HiDXS, the kinetics of inhibition of EcDXS is uncompetitive with respect to pyruvate. The $K_{\mathrm{i}}$ pyruvate value was calculated to be $75 \mu \mathrm{M}$ from a secondary plot (Supplementary Figure S2B) of the Lineweaver-Burk plot (Supplementary Figure S2A). A Lineweaver-Burk plot of the initial velocities for various concentrations of D-GAP and a fixed concentration of pyruvate $\left(50 \mu \mathrm{M}, \approx K_{\mathrm{m}}\right)$ at different concentrations of ketoclomazone is shown in Supplementary Figure S2C. As is the case with HiDXS, the four lines in the plot intersect in the second quadrant, indicating that the kinetics of inhibition is mixed type with respect to D-GAP. $K_{\mathrm{m}}^{\mathrm{D}-\mathrm{GAP}}$ and $K_{\mathrm{i}}^{\prime}{ }^{\prime}$-GAP values were calculated to be 220 and $460 \mu \mathrm{M}$, respectively, from a secondary plot (Supplementary Figure S2D) of the Lineweaver-Burk plot (Supplementary Figure S2C).

\section{DISCUSSION}

In this study, we have demonstrated for the first time that a herbicide, ketoclomazone, inhibits DXP synthase in the MEP pathway, resulting in antibacterial activity against E. coli and H. influenzae. However, its antibacterial activity was not as potent as the activities of fosmidomycin and ampicillin (Table 1), possibly because ketoclomazone was developed as a herbicide. Derivatization of ketoclomazone might improve its antibacterial activity. Mao et al. ${ }^{33}$ reported on a targetbased approach to identify possible Mycobacterium tuberculosis DXP synthase inhibitors from the structure of a known transketolase inhibitor. Ultimately they found two analogs of the transketolase inhibitor that showed antibacterial activity against M. tuberculosis with MIC values of 7.6 and $7.7 \mu \mathrm{M}$.

Kinetics of DXP synthase demonstrated the ping-pong bi bi mechanism of this enzyme (Figure 4). DXP synthase (E) first reacts with pyruvate (A) to form a Michaelis-Menten-type complex (EA) that then breaks down to yield a modified DXP synthase (F) with dissociation of $\mathrm{CO}_{2}(\mathrm{P})$ before the second substrate D-GAP (B) binds to the modified enzyme $(\mathrm{F})$. Then, the modified enzyme $(\mathrm{F})$ reacts with D-GAP (B) to form another Michaelis-Menten-type complex (FB) that finally breaks down to yield DXP synthase (E) with release of the reaction product DXP $(\mathrm{Q})$. This ping-pong bi bi mechanism is consistent with a thiamine-dependent reaction mechanism previously proposed by Rohmer et al. ${ }^{34}$ 

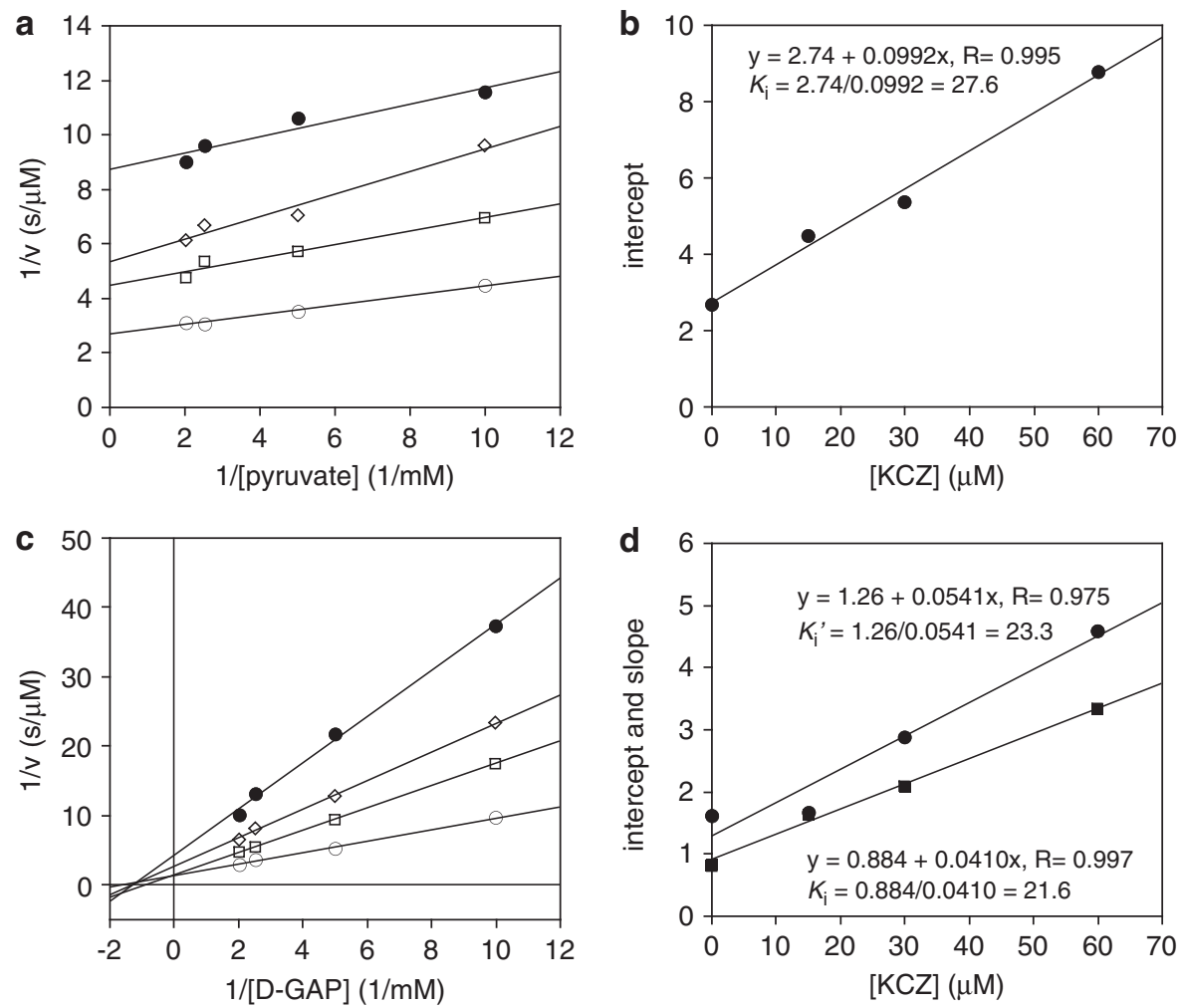

Figure 3 Kinetic analysis of ketoclomazone inhibition of $H$. influenzae 1-deoxy-D-xylulose 5-phosphate (DXP) synthase. Lineweaver-Burk plots for the inhibition of DXP synthase by ketoclomazone in the presence of (a) a fixed D-glyceraldehyde 3-phosphate (D-GAP) concentration of $0.2 \mathrm{mM}\left(\approx K_{\mathrm{m}}^{\text {pyruvate }}\right)$ or (c) a fixed pyruvate concentration of $0.2 \mathrm{~mm}\left(\approx K_{\mathrm{m}}^{\mathrm{D}-\mathrm{GAP}}\right)$ are shown. The ketoclomazone concentrations are: $0 \mu \mathrm{m}$, circle; $15 \mu \mathrm{m}, \mathrm{square} ; 30 \mu \mathrm{m}$, diamond; and $60 \mu \mathrm{m}$, filled circle. (b) Secondary plot of the Lineweaver-Burk plot (a). The linear regression formula, the correlation coefficient (R) and the formula for $K_{\mathrm{i}}$ are inserted. (d) Secondary plot of the Lineweaver-Burk plot (c). Filled square, slope; filled circle, intercept. The linear regression formulas, the correlation coefficients $(\mathrm{R})$ and the formulas for $K_{\mathrm{i}}$ and $K_{\mathrm{i}}^{\prime}$ are inserted.

$$
\begin{aligned}
& \mathrm{E}+\mathrm{A} \stackrel{K_{\mathrm{m}}^{\text {pyruvate }}}{\rightleftharpoons} \mathrm{EA} \rightleftharpoons \mathrm{FP} \stackrel{\mathrm{P}}{\longrightarrow} \mathrm{F}+\mathrm{B} \stackrel{K_{\mathrm{m}}^{\mathrm{D}-\mathrm{GAP}}}{\rightleftharpoons} \mathrm{FB} \rightleftharpoons \mathrm{EQ} \longrightarrow \mathrm{E}+\mathrm{Q} \\
& \begin{array}{llc}
+ & + & + \\
\|_{\mathrm{EAI}} K_{\mathrm{i}}^{\text {pyruvate }} & \|_{K_{\mathrm{i}}^{\mathrm{D}-\mathrm{GAP}}} & \| K_{\mathrm{i}}^{\prime \mathrm{D}-\mathrm{GAP}} \\
\mathrm{FI}+\mathrm{B} \rightleftharpoons \mathrm{FBI} &
\end{array}
\end{aligned}
$$

\begin{tabular}{lcccccc}
\hline & $K_{\mathrm{m}}{ }^{\text {pyruvate }}$ & $K_{\mathrm{i}}^{\text {pyruvate }}$ & $(\mu \mathrm{M})$ & $K_{\mathrm{m}}{ }^{\text {D-GAP }}$ & $K_{\mathrm{i}}^{\mathrm{D}-\mathrm{GAP}}$ & $K_{\mathrm{i}}^{\text {D-GAP }}(\mu \mathrm{M})$ \\
\hline H. influenzae & 190 & 28 & & 190 & 22 & 23 \\
E. coli & 48 & 75 & 370 & 220 & 460
\end{tabular}

Figure 4 Proposed mechanism of inhibition of 1-deoxy-D-xylulose 5-phosphate (DXP) synthase by ketoclomazone. The DXP synthase reaction proceeds through the ping-pong bi bi mechanism. In the DXP synthase reaction, the kinetics of inhibition by ketoclomazone is uncompetitive with respect to pyruvate and mixed type with respect to D-glyceraldehyde 3-phosphate (D-GAP). Ketoclomazone (I) cannot bind to free enzyme (E) but can bind to the EA complex, modified enzyme (F) and FB complex. The dissociation constants of ketoclomazone for binding to $H$. influenzae DXP synthase (HiDXS) and $E$. coli DXP synthase (EcDXS) are listed. Details are described in the Discussion. A, pyruvate; B, D-GAP; P, $\mathrm{CO}_{2} ; \mathrm{Q}, \mathrm{DXP}$.

We demonstrate in this study that the kinetics of inhibition by ketoclomazone is uncompetitive with respect to pyruvate and mixed type with respect to D-GAP. On the basis of the demonstration, we speculate that the inhibitory mechanism of ketoclomazone is as follows (Figure 4): first, ketoclomazone (I) tries to bind to free enzyme HiDXS
(E) but is unable to do so because uncompetitive inhibitors can bind only to the enzyme-substrate complex, and not to the free enzyme. If the first substrate, pyruvate (A), binds to the free enzyme (E) to form the HiDXS-pyruvate complex (EA), it presumably causes a conformational change in an unidentified inhibitor site different from the 
pyruvate-binding site on the HiDXS-pyruvate complex (EA), allowing ketoclomazone (I) to bind to the inhibitor site, which is remote from the active site. After release of $\mathrm{CO}_{2}(\mathrm{P})$, ketoclomazone (I) can bind to the inhibitor site on both the modified HiDXS (F) and the modified HiDXS-GAP complex (FB) to inhibit the enzyme.

We calculated dissociation constants of ketoclomazone for binding to HiDXS and EcDXS to compare the constants with each other. The three dissociation constants $(28,22$ and $23 \mu \mathrm{M})$ of ketoclomazone for binding to HiDXS were almost equal, suggesting that almost no substrate-dependent conformational change occurs in HiDXS during catalysis. By contrast, the dissociation constants (75, 220 and $460 \mu \mathrm{M})$ of ketoclomazone for binding to EcDXS significantly increased as the reaction progressed, suggesting that a substrate-dependent conformational change may occur in EcDXS. In addition, the dissociation constants of ketoclomazone for binding to EcDXS were much higher than those for binding to HiDXS. The marked difference of the dissociation constants may reflect a difference in sensitivity to ketoclomazone between $H$. influenzae and $E$. coli.

Although in this study we revealed the inhibitory mechanism of ketoclomazone on DXP synthase, we have not yet identified the inhibitor-binding site. As HiDXS and EcDXS exhibit distinct differences in sensitivity to ketoclomazone, the unidentified ketoclomazone-binding site might involve amino-acid residues that are not conserved between HiDXS and EcDXS (Supplementary Figure S3). Thus, we looked closely at the previously solved crystal structure of EcDXS, ${ }^{35}$ but we have not yet obtained information about ketoclomazone binding. Further insights into the inhibitor-binding site require crystal structures of HiDXS complexed with ketoclomazone. The structures will help in the design of ketoclomazone derivatives with more potent antibacterial activity.

\section{ACKNOWLEDGEMENTS}

We are grateful to Dr Takuya Hamaguchi and Dr Yuriko Nozawa of Taisho Pharmaceutical for measuring the MIC of the antibiotics against Haemophilus influenzae. This study was supported by the Industrial Technology Research Grant Program from the New Energy and Industrial Technology Development Organization (NEDO) of Japan (04A01538a to TK).

1 Cane, D. E. Comprehensive Natural Products Chemistry (Elsevier, Amsterdam, Netherland, 1999).

2 Kuzuyama, T. Mevalonate and nonmevalonate pathways for the biosynthesis of isoprene units. Biosci. Biotechnol. Biochem. 66, 1619-1627 (2002).

3 Kuzuyama, T. \& Seto, H. Diversity of the biosynthesis of the isoprene units. Nat. Prod. Rep. 20, 171-183 (2003).

4 Sprenger, G. A. et al. Identification of a thiamin-dependent synthase in Escherichia coli required for the formation of the 1-deoxy-D-xylulose 5-phosphate precursor to isoprenoids, thiamin, and pyridoxol. Proc. Natl Acad. Sci. USA 94, 12857-12862 (1997).

5 Lange, B. M., Wildung, M. R., McCaskill, D. \& Croteau, R. A family of transketolases that directs isoprenoid biosynthesis via a mevalonate-independent pathway. Proc. Natl Acad. Sci. USA 95, 2100-2104 (1998).

6 Lois, L. M et al. Cloning and characterization of a gene from Escherichia coli encoding a transketolase-like enzyme that catalyzes the synthesis of D-1- deoxyxylulose 5-phosphate, a common precursor for isoprenoid, thiamin, and pyridoxol biosynthesis. Proc. Natl Acad. Sci. USA 95, 2105-2110 (1998).

7 Kuzuyama, T., Takahashi, S., Watanabe, H. \& Seto, H. Direct formation of 2-C-methyl-Derythritol 4-phosphate from 1-deoxy-D-xylulose 5-phosphate by 1-deoxy-D-xylulose 5 -phosphate reductoisomerase, a new enzyme in the non-mevalonate pathway to isopentenyl diphosphate. Tetrahedron Lett. 39, 4509-4512 (1998).

8 Takahashi, S., Kuzuyama, T., Watanabe, H. \& Seto, H. A 1-deoxy-D-xylulose 5 -phosphate reductoisomerase catalyzing the formation of $2-C$-methyl-D-erythritol 4-phosphate in an alternative nonmevalonate pathway for terpenoid biosynthesis. Proc. Natl Acad. Sci. USA 95, 9879-9884 (1998).
9 Rohdich, F. et al. Cytidine 5'-triphosphate-dependent biosynthesis of isoprenoids: YgbP protein of Escherichia coli catalyzes the formation of 4-diphosphocytidyl-2-C-methylerythritol. Proc. Natl Acad. Sci. USA 96, 11758-11763 (1999).

10 Kuzuyama, T. et al. Formation of 4-(cytidine 5 '-diphospho)-2-C-methyl-D-erythritol from 2-C-methyl-D-erythritol 4-phosphate by 2 - $C$-methyl-D-erythritol 4-phosphate cytidylyltransferase, a new enzyme in the nonmevalonate pathway. Tetrahedron Lett. 41, 703-706 (2000).

11 Luttgen, H. et al. Biosynthesis of terpenoids: YchB protein of Escherichia coli phosphorylates the 2-hydroxy group of 4-diphosphocytidyl-2C-methyl-D-erythritol. Proc. Natl Acad. Sci. USA 97, 1062-1067 (2000).

12 Kuzuyama, T. et al. Studies on the nonmevalonate pathway: conversion of 4-(cytidine 5 -diphospho)-2-C-methyl-D-erythritol to its 2-phospho derivative by 4 -(cytidine 5'-diphospho)-2-C-methyl-D-erythritol kinase. Tetrahedron Lett. 41, 2925-2928 (2000).

13 Herz, S. et al. Biosynthesis of terpenoids: YgbB protein converts 4-diphosphocytidyl$2 C$-methyl-D-erythritol 2-phosphate to $2 C$-methyl-D-erythritol 2,4-cyclodiphosphate. Proc. Natl Acad. Sci. USA 97, 2486-2490 (2000).

14 Takagi, M. et al. Studies on the nonmevalonate pathway: formation of 2-C-methyl-Derythritol 2,4-cyclodiphosphate from 2-phospho-4-(cytidine 5'-diphospho)-2-C-methylD-erythritol. Tetrahedron Lett. 41, 3395-3398 (2000).

15 Hecht, S. et al. Studies on the nonmevalonate pathway to terpenes: the role of the GcpE (IspG) protein. Proc. Natl Acad. Sci. USA 98, 14837-14842 (2001).

16 Rohdich, F. et al. Studies on the nonmevalonate terpene biosynthetic pathway: metabolic role of IspH (LytB) protein. Proc. Natl Acad. Sci. USA 99, 1158-1163 (2002).

17 Kuzuyama, T., Takahashi, S. \& Seto, H. Construction and characterization of Escherichia coli disruptants defective in the yaeM gene. Biosci. Biotechnol. Biochem. 63, 776-778 (1999).

18 Kuzuyama, T., Shimizu, T., Takahashi, S. \& Seto, H. Fosmidomycin, a specific inhibitor of 1-deoxy-D-xylulose 5-phosphate reductoisomerase in the nonmevalonate pathway for terpenoid biosynthesis. Tetrahedron Lett. 39, 7913-7916 (1998).

19 Jomaa, H. et al. Inhibitors of the nonmevalonate pathway of isoprenoid biosynthesis as antimalarial drugs. Science 285, 1573-1576 (1999).

20 EINaggar, S. F. et al. Metabolism of clomazone herbicide in soybean. J. Agric. Food Chem. 40, 880-883 (1992).

21 Zeidler, J., Schwender, J., Mueller, C. \& Lichtenthaler, H. K. The non-mevalonate isoprenoid biosynthesis of plants as a test system for drugs against malaria and pathogenic bacteria. Biochem. Soc. Trans. 28, 796-798 (2000).

22 Mueller, C., Schwender, J., Zeidler, J. \& Lichtenthaler, H. K. Properties and inhibition of the first two enzymes of the non-mevalonate pathway of isoprenoid biosynthesis. Biochem. Soc. Trans. 28, 792-793 (2000).

23 Ferhatoglu, Y. \& Barrett, M. Studies of clomazone mode of action. Pesticide Biochem. Physiol. 85, 7-14 (2005).

24 Sakakibara, H. et al. Agrobacterium tumefaciens increases cytokinin production in plastids by modifying the biosynthetic pathway in the host plant. Proc. Natl Acad. Sci. USA 102, 9972-9977 (2005).

25 TenBrook, P. L. \& Tjeerdema, R. S. Comparative actions of clomazone on beta-carotene levels and growth in rice (Oryza sativa) and watergrasses (Echinochloa spp). Pest Manag. Sci. 61, 567-571 (2005).

26 Kobayashi, K. et al. Lovastatin insensitive 1, a novel pentatricopeptide repeat protein, is a potential regulatory factor of isoprenoid biosynthesis in Arabidopsis. Plant Cell. Physiol. 48, 322-331 (2007).

27 Rodriguez-Concepcion, M. The MEP pathway: a new target for the development of herbicides, antibiotics and antimalarial drugs. Curr. Pharm. Des. 10, 2391-2400 (2004).

28 Fleischmann, R. D. et al. Whole-genome random sequencing and assembly of Haemophilus influenzae Rd. Science 269, 496-512 (1995).

29 Kennedy, I. A., Hemscheidt, T., Britten, J. F. \& Spenser, I. D. 1-Deoxy-D-xylulose. Can. J. Chem. 73, 1329-1337 (1995).

30 Altincicek, B. et al. Tools for discovery of inhibitors of the 1-deoxy-D-xylulose 5-phosphate (DXP) synthase and DXP reductoisomerase: an approach with enzymes from the pathogenic bacterium Pseudomonas aeruginosa. FEMS Microbiol. Lett. 190, 329-333 (2000).

31 Kuzuyama, T., Takagi, M., Takahashi, S. \& Seto, H. Cloning and characterization of 1-deoxy-D-xylulose 5-phosphate synthase from Streptomyces sp. strain CL190 that uses both the mevalonate and nonmevalonate pathways for isopentenyl diphosphate biosynthesis. J. Bacteriol. 182, 891-897 (2000).

32 Wungsintaweekul, J. et al. Phosphorylation of 1-deoxy-D-xylulose by D-xylulokinase of Escherichia coli. Eur. J. Biochem. 268, 310-316 (2001).

$33 \mathrm{Mao}$, J. et al. Structure-activity relationships of compounds targeting Mycobacterium tuberculosis 1-deoxy-D-xylulose 5-phosphate synthase. Bioorg. Med. Chem. Lett. 18, 5320-5323 (2008).

34 Rohmer, M. et al. Glyceraldehyde 3-phosphate and pyruvate as precursors of isoprenic units in an alternative non-mevalonate pathway for terpenoid biosynthesis. J. Am. Chem. Soc. 118, 2564-2566 (1996).

35 Xiang, S. et al. Crystal structure of 1-deoxy-D-xylulose 5-phosphate synthase, a crucial enzyme for isoprenoids biosynthesis. J. Biol. Chem. 282, 2676-2682 (2007). 